\title{
A Measurement of the Gravitational Acceleration with Many Different Pendula
}

Samuele Straulino, Università di Firenze, Italia

$\mathrm{T}$ The pendulum has a great relevance in physics and it has been explored in educational papers from many theoretical or experimental points of view (see, for example, Refs. 1-12 and references therein). Here a method for the measurement of the gravitational acceleration with a large number of trials is presented; we assume that the systematic errors can be neglected. The experiment has been carried out with 250 first-year university students (students not enrolled in physics). Although the pendulum can be considered as a standard part of the introductory courses in physics laboratory, the approach described here allows students to handle various aspects of data analysis.

\section{The fixed-length pendulum}

The 250 students of the course were divided in groups of four or five. First of all, students measured the period $T$ of a fixed-length simple pendulum: they investigated how to reduce the error. Without any hint, each group was required to measure five times the period of the pendulum (time for a complete oscillation) in the best possible way, by using a digital stopwatch whose sensitivity is $0.01 \mathrm{~s}$. Usually students choose the time interval between the two subsequent ends on the same side with respect to the equilibrium position. As an example, the values measured by one of the groups are reported in Fig. 1(a) on the time axis. The same students were then required to measure the period again with the following instruction: when the swinging bob crosses the equilibrium position, start timing and count 10 periods, finally dividing the overall time by 10 . Data obtained by the same group with this method are reported in Fig. 1(b).

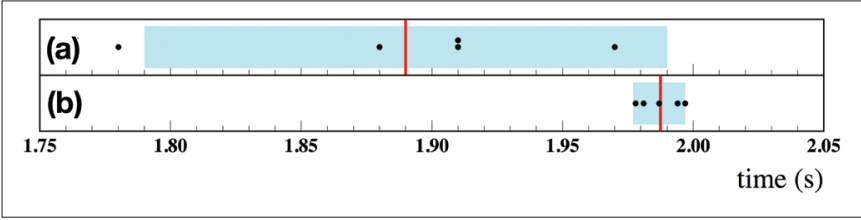

Fig. 1. Results of two sets of measurements for the period of a simple pendulum. Red lines mark the mean values.

In the figure, the red line indicates the mean value $T_{\mathrm{m}}$ for each set of data. Blue strips are the regions corresponding to $T_{\mathrm{m}} \pm \Delta T$. The uncertainty $\Delta T$ is estimated as $\left(T_{\mathrm{MAX}}{ }^{-}\right.$ $\left.T_{\mathrm{MIN}}\right) / 2$, with the result being about $0.1 \mathrm{~s}$ for the first set of data and $0.01 \mathrm{~s}$ for the other one.

Students could clearly observe that values are much less scattered in the second set. In the latter method, only $1 / 10$ of the personal reaction time (an error larger than the sensitivity of the stopwatch) affects the period: this is the main advantage. Furthermore (with smaller benefit on the uncertainty) the stopwatch should be started and stopped when the bob speed has a maximum, in the central point of the oscillation (with the reference of a vertical bar). Conversely, it is not easy to capture the instant at which the bob stops before reversing the motion.

After this preliminary training, many students showed a good ability in timing; only a minority (approximately 10\%) still had difficulties and required some additional hints or explanations. Thereafter students used this procedure for measuring the period in order to reduce uncertainties. In principle students may repeat the measurements by using the same fixed-length pendulum, but with different bob masses and different amplitudes, to discover that, within the experimental errors, the period does not change.

\section{The variable-length pendulum}

A nylon thread (fishing line), approximately $1 \mathrm{~m}$ long, was prepared for each group: it was fastened to a high enough support, leaning on a table. The oscillating mass is composed of a bolt, a nut, and two thick square washers

(Fig. 2), and can be positioned along the thread. In this way, students can make an arbitrary long pendulum.

Each group was required to

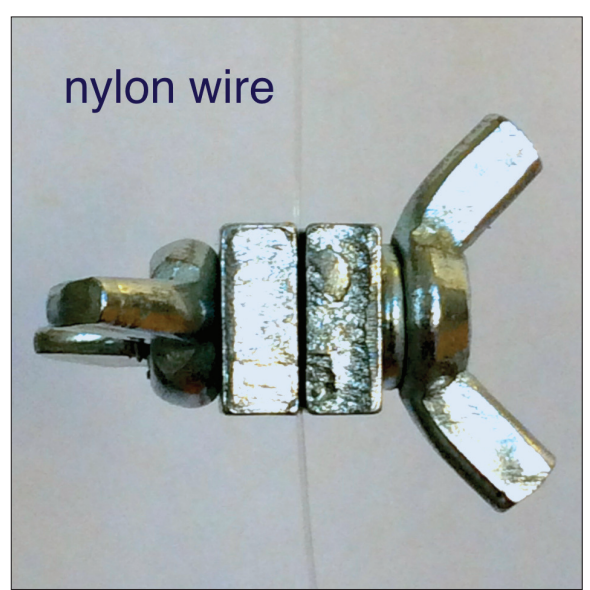

Fig. 2. Picture of the bolt that students used to make a simple pendulum; it was hanging from a nylon thread. The washer is $4 \mathrm{~mm}$ thick and $10 \mathrm{~mm}$ wide. Students determined approximately (by symmetry) the position of the center of mass and measured the length of the pendulum from the beginning of the thread to that point. make five different pendula, by placing the mass at different positions along the thread, and to measure length (with a folding rule whose sensitivity is $1 \mathrm{~mm}$ ) and period (with a digital stopwatch) of each one.

The aim of the experiment is to obtain a measurement of the gravitational acceleration $g$ by using the formula of the pe$\operatorname{riod} T$ for small oscillations of a simple pendulum:

$$
T=2 \pi \sqrt{l / g} .
$$


Students were warned to work in the "small oscillation regime" (they were required to maintain an angular amplitude smaller than $10^{\circ}$ ). Probably, the prescription has not been satisfied in some cases; however we expect a small effect on the result. ${ }^{13}$ Time measurements were taken only once (after the training performed in the previous steps with the fixed-length pendulum), but each time included 10 periods. As a rough $a$ priori estimation of the uncertainties, we assumed $\pm 0.1 \mathrm{~cm}$ on the length of the pendulum (this is the sensitivity error of the rule) and $\pm 0.01 \mathrm{~s}$ on the period (because on the complete measurement, covering 10 periods, the estimated error is $\pm 0.1 \mathrm{~s}$, as discussed before).

As an example, the measurements taken by one of the groups are reported in Table I.

Table I.

\begin{tabular}{|l|l|l|l|l|l|}
\hline $\boldsymbol{L}$ (cm) & 26.3 & 33.4 & 46.7 & 57.1 & 76.1 \\
\hline $\boldsymbol{T}$ (s) & 1.01 & 1.15 & 1.37 & 1.51 & 1.74 \\
\hline
\end{tabular}

\section{Data analysis}

After all the groups collected their data, the students and the teacher merged values together to obtain a cumulative measurement of $g$. The value of the gravitational acceleration was calculated with a computer spreadsheet for all the pairs of values $\left(L_{i}, T_{i}\right)$. The distribution is shown in Fig. 3: a few values of $g$ exhibit a large discrepancy from the expected value $(\Delta g / g>10 \%)$.

Let us assume that our values follow a Gaussian distribution $G(x)$. When $G(x)$ is normalized to 1 and centered in $x=0$, the probability to obtain any value within a distance $l$ from the center is given by the integral

$$
p(l)=\int_{-l}^{l} G(x) d x .
$$

The expected events in this range are $n_{i}=N p(l), N$ being the number of measurements, and $n_{e}=N[1-p(l)]$ are expected outside the interval. We apply Chauvenet's criterion ${ }^{16}$ to the data sample to check if some value must be rejected. According to Chauvenet, we reject a suspect value if $n_{e} \leq 0.5$ (no more than 0.5 events as deviant as the suspect are expected). If $n_{e}=$ 0.5 and $N=280$, the above equation gives $p(l)=0.9982$ : this probability corresponds to $l=3.1 \sigma$, as obtained from tabulated integrals of the Gaussian function. In our case we found nine measurements that are placed beyond such limit (red bars in Fig. 3) and these values have been rejected. No further data rejection is applied: according to a common practice, the Chauvenet's criterion should be applied only once. ${ }^{16}$ With the surviving 271 values, the histogram's mean is $9.81 \mathrm{~m} / \mathrm{s}^{2}$ and the standard deviation is $0.28 \mathrm{~m} / \mathrm{s}^{2}$. This has the following statistical meaning: choosing a student in the sample to perform another measurement, $68 \%$ is the probability to find the new value of $g$ within a standard deviation around the mean. However the error on the mean value of the distribution is $\sigma / \sqrt{N} \cong 0.02 \mathrm{~m} / \mathrm{s}^{2}$. The average has greater precision than any other measurement, being obtained from all measured values.

The data are then reported on a graph, where the squared period $T^{2}$ is given as a function of $4 \pi^{2} L$ (Fig. 4 ). In this repre-

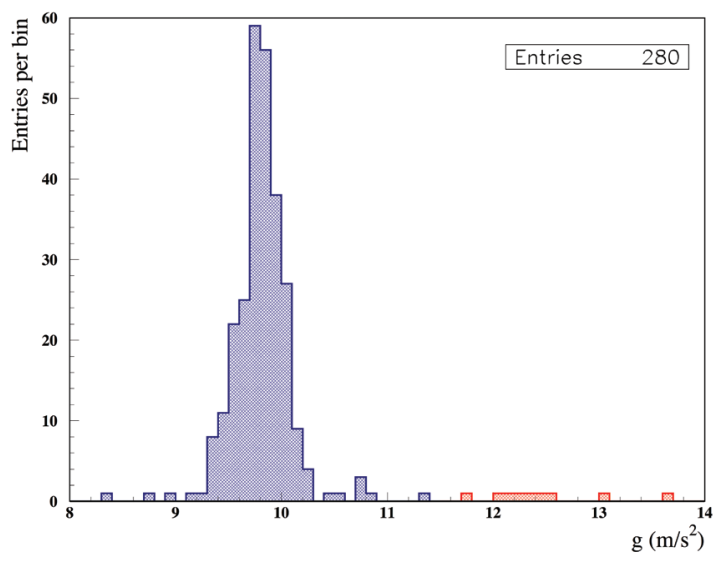

Fig. 3. Distribution of 280 values of $g$ obtained with different pendulum lengths. The bin width of the histogram $\left(0.1 \mathrm{~m} / \mathrm{s}^{2}\right)$ was chosen according to the Freedman-Diaconis rule. ${ }^{15}$ Some bins, marked in red, contain data that were later rejected following Chauvenet's criterion, as explained in the text.

sentation, the points should follow a straight line from the origin with angular coefficient $1 / g$. The orange and blue straight lines in the figure show the effects of assigning errors to the measured quantities. Suppose you have a set of points $\left(L_{i}, T_{i}\right)$ placed exactly on the straight line

$$
T^{2}=\left(4 \pi^{2} L\right) / g \text {. }
$$

When the length $L_{i}$ varies in the range $L_{i} \pm 0.3 \mathrm{~cm}$ (three times the $a$ priori estimated error), the corresponding point is displaced from its position and moves horizontally within the (narrow) band delimited by the blue straight lines. Similarly, when the period $T_{i}$ varies in the range $T_{i} \pm 0.03 \mathrm{~s}$ (again three times the estimated error), the corresponding point moves vertically within the band delimited by the orange straight lines. From the picture, the effect of the time error on the angular coefficient seems to be predominant.

Red points in the graph correspond to the discarded measurement and they are not included in the calculation of $g$. From Fig. 4 we see that all red points have too small values of $T$. Probably this can be explained with a quite common wrong procedure: sometimes students count nine periods instead of 10. In fact, if the time values of the red points are multiplied by $10 / 9$, they move on the "main sequence" of the other points.

We can calculate the best interpolating straight line of the form $y=k x$. When

$$
\frac{\Delta x}{x} \ll \frac{\Delta y}{y}
$$

and the uncertainties in $y$ all have the same magnitude (unweighted fit), the angular coefficient of the fitting straight line can be obtained by minimizing the function $F(k)$ defined as:

$$
F(k)=\sum_{i=1}^{N}\left[\left(y_{i}-k x_{i}\right)^{2}\right]
$$

When the uncertainties $\sigma_{i}$ on $y$ are different (weighted fit), the associated function $F(k)$ is slightly more complicated ${ }^{17}$ :

$$
F(k)=\sum_{i=1}^{N} \frac{\left(y_{i}-k x_{i}\right)^{2}}{\sigma_{i}^{2}}
$$




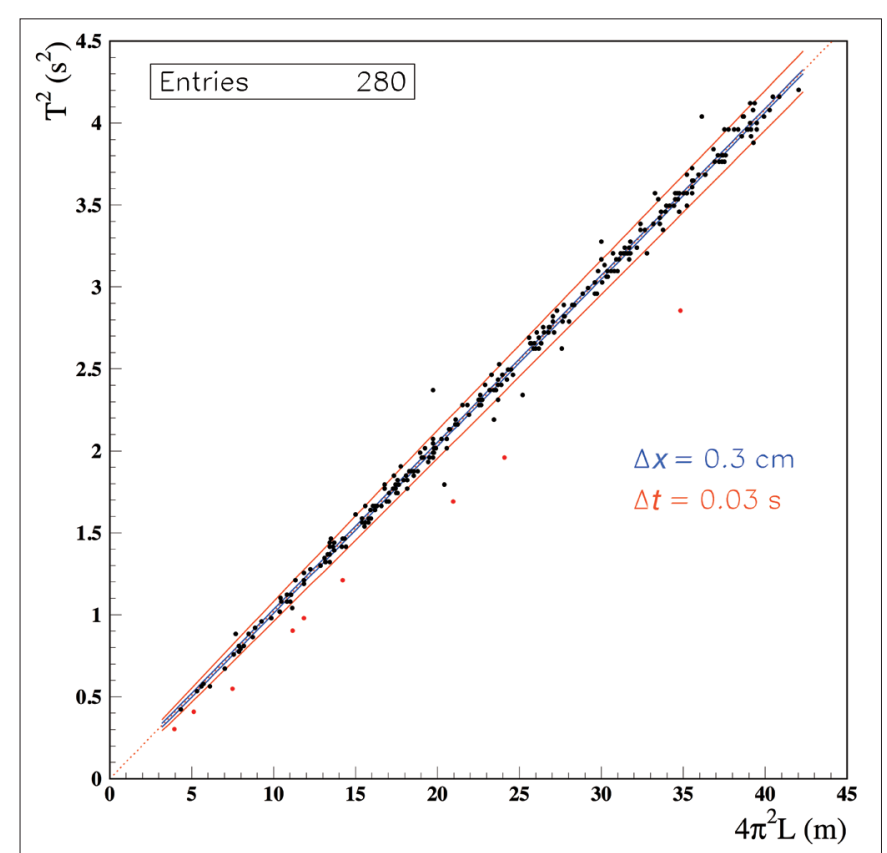

Fig. 4. Graph where the squared period $T^{2}$ is reported as a function of $4 \pi^{2} L$ for all the measured values. The red dashed line is the result of the fit.

To account for uncertainties in both time and distance, we introduced the equivalent error $\sigma_{i}^{2}=\left(\Delta y_{i}\right)^{2}+\left(k \Delta x_{i}\right)^{2}$, as suggested in Ref. 14. Here an approximated value can be used for $k$. We computed the value of $k$ for which $F(k)$ has a minimum. The result for the reciprocal of the slope is:

$$
1 / k=(9.80 \pm 0.02) \frac{\mathrm{m}}{\mathrm{s}^{2}} .
$$

This is our measurement of the gravitational acceleration $g$ and it is in agreement with what is expected ${ }^{18}$ for the latitude $44^{\circ} \mathrm{N}\left(g=9.805 \mathrm{~m} / \mathrm{s}^{2}\right)$, where the experiment was carried out.

\section{Concluding remarks}

The experiment is simple enough to be within the grasp of a first-year university student. Also the experimental apparatus is very simple and can be replicated on several workstations for courses that have many students. The final result is obtained through data processing that allows students to review the Gaussian statistics and the criteria for data rejection.

\section{Acknowledgments}

The author is indebted to Prof. Massimo Bongi for useful suggestions about this article.

\section{References}

1. S. Li and S. Feng, "Precision measurement of the period of a pendulum using an oscilloscope," Am. J. Phys. 35, 1071-1073 (Nov. 1967).
2. R. A. Nelson, "The pendulum - Rich physics from a simple system," Am. J. Phys. 54, 112-121 (Feb. 1986).

3. D. P. Jackson, "Rendering the 'not-so-simple' pendulum experimentally accessible," Phys. Teach. 34, 86-89 (Feb. 1996).

4. D. P. Randall, "Student-friendly precision pendulum," Phys. Teach. 37, 390-393 (Oct. 1999).

5. A. Dupré and P. Janssen, "An accurate determination of the acceleration of gravity $g$ in the undergraduate laboratory," Am. J. Phys. 68, 704-711 (Aug. 2000).

6. T. Lewowski and K. Wozniak, "The period of a pendulum at large amplitudes: A laboratory experiment," Eur. J. Phys. 23, 461-464 (2002).

7. M. Vannoni and S. Straulino, "Low-cost accelerometers for physics experiments," Eur. J. Phys. 28, 781-787 (2007).

8. J. Sinacore and H. Takai, "Measuring $g$ using a magnetic pendulum and telephone pickup," Phys. Teach. 48, 448-449 (Oct. 2010).

9. Khairurrijal, E. Widiatmoko, W. Srigutomo and N. Kurniasih, "Measurement of gravitational acceleration using a computer microphone port," Phys. Educ. 47, 709-714 (2012).

10. J. Briggle, "Analysis of pendulum period with an iPod touch/ iPhone," Phys. Educ. 48, 285-288 (2013).

11. V. Oliveira, "Measuring $g$ with a classroom pendulum using changes in the pendulum string length," Phys. Educ. 51, 063007 (2016).

12. L. A. Ladino and H. S. Rondón, "Determining the damping coefficient of a simple pendulum oscillating in air," Phys. Educ. 52,

13. The period of the pendulum is $T=2 \pi \sqrt{\frac{l}{g}}\left(1+\theta^{2} / 16+\cdots\right)$,
where $\theta$ is the maximum angular displacement from the equilibrium position. For $\theta=10^{\circ}=0.17$ $\mathrm{rad}$, the second term in the parentheses is about 0.002 . The departure from isochronism can be perceived when the angle is wide enough, as in the student lab reported in Ref. 14 .

14. N. G. Holmes and D. A. Bonn, "Quantitative comparisons to promote inquiry in the introductory physics lab," Phys. Teach. 53, 352-355 (Sept. 2015).

15. D. Freedman and P. Diaconis, "On the histogram as a density estimator: $L_{2}$ Theory," $Z$. Wahrscheinlichkeitstheorie verw. Gebiete 57, 453-476 (1981).

16. J. R. Taylor, An Introduction to Error Analysis (University Science Books, Sausalito, 1982).

17. I. G. Hughes and T. P. A. Hase, Measurements and their Uncertainties (Oxford University Press, New York, 2010).

18. Department of Defense World Geodetic System 1984, NIMA TR8350.2, 3rd ed., Table 3.4, Eq. 4-1.

Samuele Straulino obtained a degree in physics (1999) and a PhD in physics (2003). His background is in experimental cosmic-ray physics. Since 2004 he has been involved in a project for education in science developed by Florence University. He has trained many teachers of primary and secondary schools at the Florence University. samuele.straulino@unifi.it 